\title{
SEVERE IDIOPATHIC POSTERIOR SCLERITIS IN CHILDREN
}

\author{
W. H. WOON, M. R. STANFORD and E. M. GRAHAM \\ London
}

\begin{abstract}
SUMMARY
Posterior scleritis is an uncommon condition that usually occurs in adults and the diagnosis is often missed. It is even more rare in children. We report five cases of the disease in children. The patients presented with severe pain in an inflamed eye and usually had orbital signs with lid swelling and limitation of extraocular movements; four of the five had optic disc swelling and two had exudative retinal detachments. The diagnosis was confirmed on B-scan ultrasonography and CT scanning and no child had clinical or laboratory evidence of associated systemic disease. The disease took a protracted course and all the children required long-term systemic immunosuppression (15-27 months), which was well tolerated. All children retained good vision and were either off treatment or on a small dose of immunosuppression at last follow-up. This condition, although rare, should be recognised in children as part of the differential diagnosis of acute orbital inflammation.
\end{abstract}

Posterior scleritis is an unusual condition that is most commonly seen in middle-aged women. ${ }^{1,2}$ The protean manifestations and the range of severity of the condition can lead to delay in diagnosis and subsequent loss of vision. Its occurrence in children is rare, with only 14 cases reported in the literature. ${ }^{1-6}$ Reported cases have ranged in severity from mild cases treated with non-steroidal anti-inflammatory agents to cases with visual loss due to optic atrophy. We report a further 5 children with severe posterior scleritis who required treatment with high-dose immunosuppression.

\section{PATIENTS AND METHODS}

Five children were diagnosed as having posterior scleritis over a 9 month period at the Medical Eye Unit at St Thomas' Hospital. All patients underwent a complete ophthalmic and general examination with

Correspondence to: E. M. Graham, Medical Eye Unit, St Thomas' Hospital, Lambeth Palace Road, London SE1 7EH, UK.
B-scan ultrasonography and CT scan of the orbits. A chest radiograph and blood tests including a full blood count, liver function tests, erythrocyte sedimentation rate, C-reactive protein, antinuclear antibody and anti-neutrophil cytoplasmic antibody were performed.

\section{CASE REPORTS}

Case 1

A 14-year-old West Indian boy presented with a 2 week history of a sore right eye with swollen eyelids following an upper respiratory tract infection. On examination visual acuity was $6 / 6$ in each eye; the left eye was quiet and the right eye was injected with $1+$ cells in the anterior chamber. The eyelids were swollen and the conjunctiva oedematous. There was limitation of ocular movement of the right eye in all directions of gaze, particularly on upgaze. Results of funduscopy and a general examination were normal.

An initial diagnosis of orbital cellulitis was made and the patient was treated with intravenous amoxycillin, flucloxacillin and metronidazole. An orbital CT scan showed clear paranasal sinuses but marked scleral thickening of the right eye. This was confirmed by B-scan ultrasonography and a diagnosis of posterior scleritis was made. All investigations were negative including viral and toxoplasma titres. The antibiotics were stopped and the patient was started on oral prednisolone $60 \mathrm{mg}$ once daily $(1 \mathrm{mg} /$ $\mathrm{kg}$ ) and ranitidine $150 \mathrm{mg}$ twice daily with rapid improvement.

The patient was discharged home on $40 \mathrm{mg} / \mathrm{day}$ prednisolone but returned the next day complaining of pain in both eyes. On examination at that time visual acuity was $6 / 9$ in each eye and both eyes showed $2+$ cells in the anterior chamber. The prednisolone was increased back to $60 \mathrm{mg} / \mathrm{day}$ and then reduced in a stepwise fashion at weekly intervals to $40 \mathrm{mg} / \mathrm{day}$ and then $30 \mathrm{mg} / \mathrm{day}$. After the third week of prednisolone there was still a mild 
anterior uveitis in both eyes and bilateral disc swelling was now present.

The patient was referred to the Medical Eye Unit and cyclosporin A was started at a dose of $5 \mathrm{mg} / \mathrm{kg}$. The discs subsequently returned to normal. The patient was taken off both cyclosporin A and prednisolone 15 months after presentation and ibuprofen was substituted. The visual acuity was $6 / 4$ unaided in either eye and the eyes were quiet with no limitation of ocular movement.

\section{Case 2}

A 12-year-old Indian boy complained of a progressively red and painful right eye for 10 days. The patient was otherwise fit and well. On examination visual acuity was $6 / 4$ in each eye with swelling of the right eyelids. There was a mild anterior uveitis on the right and an area of scleritis was identified temporarily in the right eye.

The patient was treated with topical Betnesol and mydriatics, but on review 2 days later the pain and swelling around the right eye had increased, particularly on eye movement, with diplopia in all positions of gaze. Visual acuity remained at $6 / 6$ in each eye but the patient had developed swelling of the right optic disc. A B-scan ultrasound showed thickening of the sclera in the right eye and a diagnosis of posterior scleritis was made and the patient started on oral prednisolone $60 \mathrm{mg}$ once daily $(1.5 \mathrm{mg} / \mathrm{kg})$ and ranitidine $150 \mathrm{mg}$ twice daily. General examination and serum investigations were negative. A CT scan of the orbits confirmed scleral thickening in the right eye. There was a rapid improvement in the patient's symptoms on steroids and after 4 days the dose was reduced to $40 \mathrm{mg}$ once daily.

Within 2 days of this reduction the patient relapsed and the prednisolone had to be increased back to 60 $\mathrm{mg} /$ day. Over the next 2 weeks two further relapses occurred on attempts to reduce the prednisolone below $30 \mathrm{mg} / \mathrm{day}$; the patient was subsequently referred to the Medical Eye Unit and cyclosporin A was started at a dose of $5 \mathrm{mg} / \mathrm{kg}$. Both treatments were tailed off over a year and the final visual acuity was $6 / 6$ in each eye with full ocular movement and no evidence of disease.

\section{Case 3}

An 11-year-old West Indian boy presented with pain and redness of the left eye. On examination vision was $6 / 5$ right, $6 / 9$ left; the right eye was quiet but the left eye was injected with anterior uveitis and anterior scleritis. Funduscopy was normal in both eyes.

The patient was started on topical steroids and mydriatics and ibuprofen, but on review 3 days later the anterior uveitis had become more severe with 3+ cells in the anterior chamber. $\mathrm{He}$ was given subconjunctival steroid and the topical steroids were increased. Three days later the patient was reviewed and vision in the left eye was reduced to $6 / 60$, there was $2+$ vitreous activity and funduscopy showed gross venous dilatation, disc swelling and peripapillary serous retinal detachment.

Treatment was continued with topical dexamethasone and ibuprofen for another 2 weeks until the right eye became affected. Visual acuity was now $2 / 60$ right, counting fingers left, there was anterior chamber activity in both eyes and funduscopy showed bilateral disc swelling and bilateral serous retinal detachments. The patient was started on prednisolone $60 \mathrm{mg} /$ day, a B-scan ultrasound showed thickening of the posterior sclera of both eyes and a diagnosis of bilateral posterior scleritis was made (Fig. 1). Within 2 days of starting high-dose systemic steroids the visual acuity had improved to $6 / 24$ right and $6 / 36$ left. The patient was transferred for further management.

No associated ocular or systemic disease was found and the results of investigations were normal. A CT scan of the orbits showed loss of definition of the posterior sclera. The visual acuity continued to improve with resolution of the serous retinal detachments and the prednisolone was gradually reduced. After 6 weeks vision had returned to $6 / 6$ in either eye and the fundus appearance returned to normal. The prednisolone was finally stopped after 9 months; two minor relapses occurred during this time. The first resolved when the prednisolone was increased from $5 \mathrm{mg}$ on alternate days back to $5 \mathrm{mg}$ once a day; the second occurred 8 months after stopping prednisolone and the patient was managed on ibuprofen $50 \mathrm{mg}$ three times a day alone. After 17 months of follow-up the visual acuity was $6 / 4$ right, $6 / 6$ left, and both eyes were quiet with the patient still on a low dose of ibuprofen.

\section{Case 4}

A 12-year-old Caucasian girl presented with a sore red left eye. A diagnosis of anterior uveitis was made

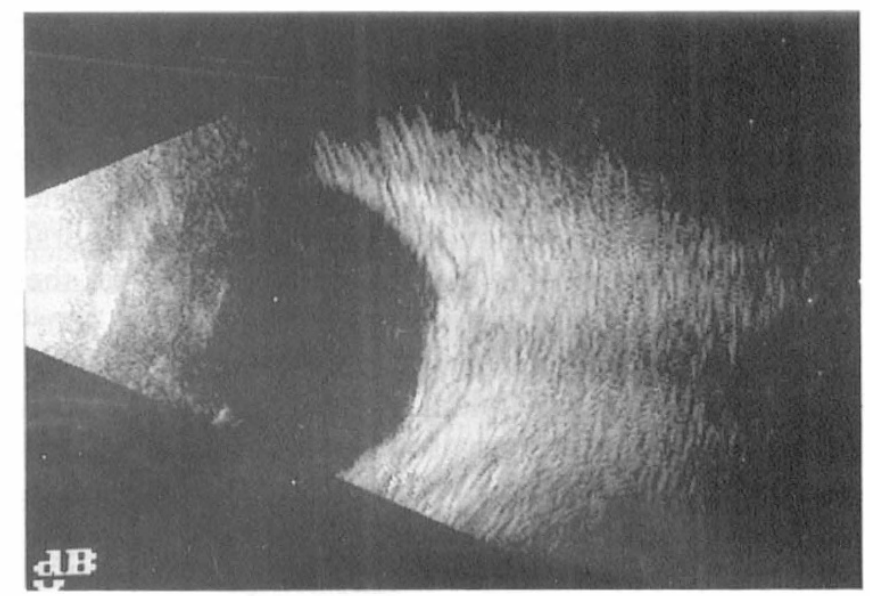

Fig. 1. Case 3. B-mode ultrasonography of the right eye. Marked scleral thickening is evident. 
and the patient was treated with topical steroids. After 2 days the eye was still red and becoming increasingly painful, and at this time visual acuity was $6 / 6$ right, 6/9 left. The eyelids were swollen and tender with evidence of a moderate anterior uveitis on the left but normal funduscopy. Past medical history and general examination were normal and investigations showed no associated systemic disease. The patient was continued on topical drops to the left eye and also received a short course of oral prednisolone (30 mg once a day, reducing to $5 \mathrm{mg}$ over 4 days). This failed to control the patient's symptoms and she developed diplopia on right and left gaze. She was subsequently transferred for further management.

An ultrasound scan showed scleral thickening of the left eye and the patient was initially commenced on ibuprofen $25 \mathrm{mg}$ three times a day. After 10 days of this treatment there was little change in the patient's condition. A CT scan of the orbit showed loss of definition of the posterior margin of the left eye. The ibuprofen was stopped and prednisolone 30 $\mathrm{mg}$ once a day $(1 \mathrm{mg} / \mathrm{kg})$ and ranitidine were initiated. The patient slowly improved and after 2 weeks the prednisolone was reduced to $40 \mathrm{mg}$ on alternate days. Unfortunately, one day after this reduction the pain in the left eye returned and this was controlled by increasing the steroids to $40 \mathrm{mg} /$ day. Over the next 3 weeks two further attempts were made to reduce the patient's systemic steroids, both times resulting in relapse of her eye condition. As she was becoming cushingoid and her weight had increased by $10 \mathrm{~kg}$ she was started on cyclosporin A at $5 \mathrm{mg} / \mathrm{kg}$. Subsequently she has had four further relapses, each one of which has responded to a mild increase in her prednisolone dosage. At 24 months of follow-up the patient still required prednisolone $8 \mathrm{mg}$ once a day and cyclosporin A $100 \mathrm{mg}$ once a day to control her disease. Her cushingoid appearance had resolved and the visual acuity returned to $6 / 5$ in each eye.

\section{Case 5}

A 9-year-old Indian girl presented with an intermittently sore red right eye for 2 months that had not responded to topical antibiotics. On examination she was noted to have minimal injection of the temporal conjunctiva in the right eye and mild swelling of the right lids. The patient returned 2 weeks later complaining that the right eye was once more painful and she had intermittent diplopia, particularly on looking to the left. She was otherwise in good health and there was no past relevant medical or ocular history.

On examination visual acuity was $6 / 9$ right, $6 / 6$ left. The right upper lid was swollen with painful restriction particularly of horizontal and upward gaze. The eyes were quiet and funduscopy showed

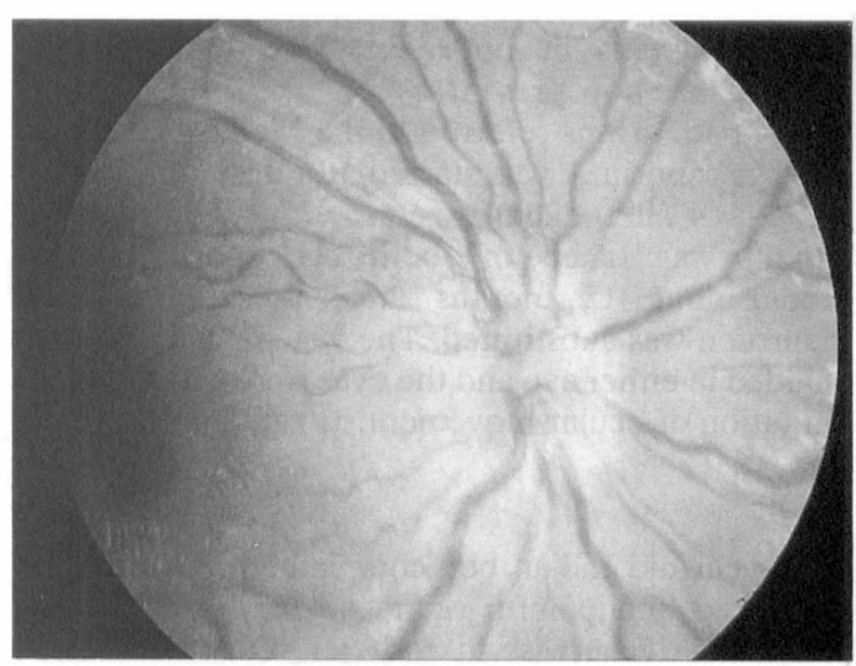

Fig. 2. Case 5. Swollen right optic disc.

a swollen right optic disc (Fig. 2). The patient was initially felt to have a right third nerve palsy. A CT scan of the orbit showed thickening of the sclera in the right eye and the diagnosis was changed to posterior scleritis (Fig. 3). The scleral thickening was confirmed by B-scan ultrasound scan. Her general examination and tests showed no evidence of associated systemic disease. The patient was started on prednisolone $30 \mathrm{mg}$ twice daily $(2 \mathrm{mg} / \mathrm{kg})$ and she responded rapidly to this treatment. The dose of prednisolone was subsequently slowly reduced and the ocular movements and the right optic disc appearance returned to normal.

After 5 months the patient's condition relapsed when the prednisolone had been reduced to $10 \mathrm{mg}$ and $5 \mathrm{mg}$ on alternate days. The right eye once more became injected with swelling of the lids, and the visual acuity in this eye fell to $6 / 36$. There was $2+$ anterior chamber cells in the right eye and the right disc had become swollen with serous elevation of the right macula. The prednisolone was increased to 30 $\mathrm{mg}$ /day. After 5 days there was no substantial improvement and the prednisolone was increased to

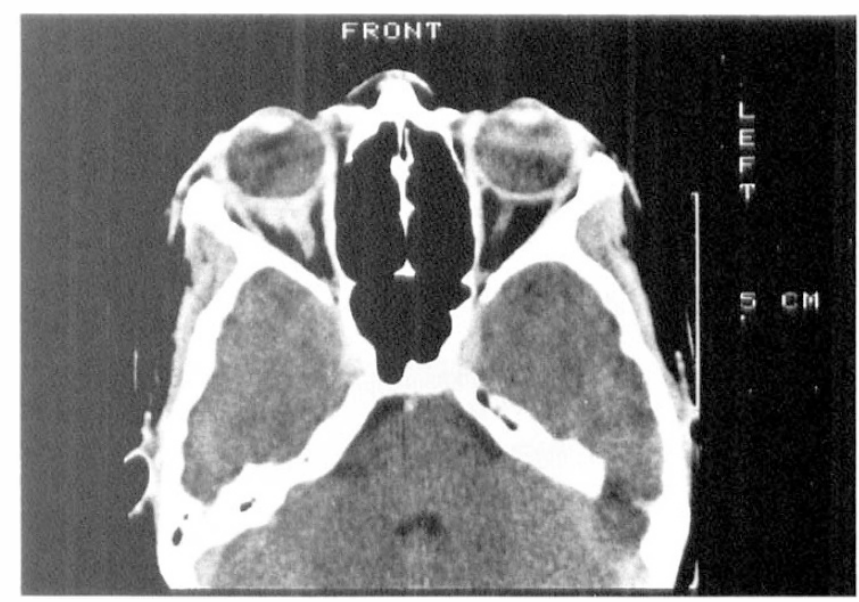

Fig. 3. Case 5. CT scan showing scleral thickening in the right eye. 
$60 \mathrm{mg} /$ day and cyclosporin A $(5 \mathrm{mg} / \mathrm{kg})$ was started. The pain and redness of the eye improved, and there was a reduction in serous elevation at the right macula with gradual improvement of the patient's visual acuity. The prednisolone was reduced in stages and 5 weeks later the vision in the affected right eye had improved to 6/18. However, the cyclosporin A was stopped because of hypertension and proteinuria. By this stage the patient had also become markedly cushingoid and her weight had increased by $10 \mathrm{~kg}$. Her treatment was changed to prednisolone $40 \mathrm{mg}$ on alternate days, azathioprine $25 \mathrm{mg}$ three times daily, ibuprofen $50 \mathrm{mg}$ twice daily, and ranitidine $150 \mathrm{mg}$ twice daily. Two weeks later the patient relapsed after the prednisolone had been reduced to $30 \mathrm{mg}$ on alternate days and increasing alternate day prednisolone failed to control this. At this time there was evidence of further swelling of the right optic disc and a further serous detachment of the right macula. A urinary tract infection was diagnosed which responded well to appropriate antibiotics. The azathioprine and ibuprofen were tailed off and the patient was re-started on cyclosporin A with daily doses of prednisolone. This brought the condition under control and after 2 weeks the visual acuity had improved to $6 / 12$ in the right.

Since then the patient has been followed up for a further 9 months and the re-introduction of cyclosporin A has allowed the prednisolone to be gradually reduced with only minor relapses. She is currently taking cyclosporin A $75 \mathrm{mg}$ twice daily, prednisolone $10 \mathrm{mg}$ once on alternate days, and topical steroids as necessary. Visual acuity remained at $6 / 9$ right, 6/6 left, and the fundal signs had resolved.

\section{Summary of Cases}

The clinical results of the patients are summarised in Table I. Five children ( 3 boys, 2 girls) with posterior scleritis with a mean follow-up of 15.8 months (range 11-23 months) are reported. The mean age of presentation was 11.6 years (range 9-14 years). Two children were West Indian, 2 Indian and 1 Caucasian.

All patients presented with severe pain in their eye but were otherwise fit and well. The condition was unilateral at presentation in all cases; 1 case soon became urequivocally bilateral and 2 further cases developed inflammatory changes in the contralateral eye. At presentation 4 of 5 cases had an anterior uveitis, swelling of the eyelids and painful limitation of ocular movement. Disc swelling was present in 1 case on presentation and developed later in 3 further cases. Visual acuity was $6 / 9$ or better at presentation in all cases. The initial diagnosis was anterior uveitis in 2 patients, orbital cellulitis in 1 , third nerve palsy in 1 , and posterior scleritis in 1 . General examination and serological testing showed no evidence of an associated systemic disease. Severe visual loss developed in 3 eyes in 2 cases due to serous macula detachment.

All patients required treatment with high-dose oral prednisolone; 4 patients required the addition of cyclosporin $\mathrm{A}$, which was well tolerated but was stopped temporarily in 1 case due to the development of proteinuria. In this latter case this was subsequently attributed to a urinary tract infection and the patient was re-started on cyclosporin $\mathrm{A}$ without further side effects and good control of her disease.

\section{DISCUSSION}

Posterior scleritis is a rare condition that can present with a wide range of symptoms and signs. It can mimic orbital conditions, optic neuritis, acute glaucoma and central serous retinopathy. ${ }^{1,2}$ For these reasons the condition is difficult to diagnose or diagnosis may be made late in the course of the disease. The disease usually presents in middle age and there are few reports of its occurrence in children. We report a series of children with posterior scleritis who presented with severe pain

Table I. Summary of cases

\begin{tabular}{|c|c|c|c|c|c|c|}
\hline Case no. & $\begin{array}{c}\text { Age } \\
\text { (years) }\end{array}$ & $\begin{array}{l}\text { Ethnic } \\
\text { origin }\end{array}$ & $\begin{array}{l}\text { Diagnosis at } \\
\text { presentation }\end{array}$ & $\begin{array}{l}\text { Predominant } \\
\text { ocular features }\end{array}$ & Treatment & Outcome \\
\hline 1 & 14 & W. Indian & Orbital cellulitis & $\begin{array}{l}\text { AAU, limited EOM } \\
\text { disc swelling }\end{array}$ & $\begin{array}{l}\text { Prednisolone, } \\
\text { cyclosporin A, } \\
\text { ibuprofen }\end{array}$ & $\begin{array}{l}\text { 6/4 6/4 Quiet } \\
\text { No treatment }\end{array}$ \\
\hline 2 & 12 & Indian & Posterior scleritis & $\begin{array}{l}\text { AAU, anterior } \\
\text { scleritis, limited } \\
\text { EOM, disc swelling }\end{array}$ & $\begin{array}{l}\text { Prednisolone, } \\
\text { cyclosporin A }\end{array}$ & 6/6 6/6 Quiet \\
\hline 3 & 11 & W. Indian & Anterior uveitis & $\begin{array}{l}\text { AAU, anterior } \\
\text { scleritis, disc } \\
\text { swelling SRD vitritis }\end{array}$ & $\begin{array}{l}\text { Prednisolone, } \\
\text { ibuprofen }\end{array}$ & $\begin{array}{l}\text { 6/4 6/6 Quiet } \\
\text { Ibuprofen }\end{array}$ \\
\hline 4 & 12 & Caucasian & Anterior uveitis & AAU, limited EOM & $\begin{array}{l}\text { Ibuprofen, } \\
\text { prednisolone, } \\
\text { cyclosporin A }\end{array}$ & $\begin{array}{l}6 / 56 / 5 \text { Quiet } \\
\text { Pred } 8 \mathrm{mg} \\
\text { Cy A } 100 \mathrm{mg}\end{array}$ \\
\hline 5 & 9 & Indian & III nerve palsy & $\begin{array}{l}\text { Limited EOM, disc } \\
\text { swelling, SRD }\end{array}$ & $\begin{array}{l}\text { Prednisolone, } \\
\text { cyclosporin A, } \\
\text { ibuprofen, } \\
\text { azathioprine }\end{array}$ & $\begin{array}{l}\text { 6/9 6/6 Quiet } \\
\text { Pred } 10 \mathrm{mg} \\
\text { Cy A } 50 \mathrm{mg}\end{array}$ \\
\hline
\end{tabular}

$\overline{\text { AAU, acute anterior uveitis; EOM, extraocular movements; SRD, serous retinal detachment; Pred, prednisolone; Cy A, cyclosporin A. }}$ 
in an inflamed eye. They usually had orbital signs with lid swelling and limitation of extraocular movements. The children were otherwise well and there were no serological markers of associated systemic disease. The diagnosis was suspected clinically initially in only 1 case but was confirmed in all cases by B-scan ultrasonography and CT scanning.

There are few published cases of posterior scleritis in children. The West Indian cohort of posterior scleritis in young adults reported by Calthorpe et al. ${ }^{1}$ had severe disease with a combination of anterior and posterior scleritis. Treatment with prednisolone was required in 4 of 5 cases. However, the patients were diagnosed late and permanent visual loss occurred in 2 cases due to optic atrophy from prolonged optic nerve swelling. Wald et al. ${ }^{3}$ have published a series of 4 cases of posterior scleritis in adolescent boys who presented with visual loss and exudative retinal detachment. Pain and anterior segment inflammatory signs were either mild or absent in 3 cases and high-dose prednisolone was not required. The remaining case seemed more acute and presented with pain and redness of the eye with mild proptosis, and in this last case high-dose systemic steroids were required. Other authors have included cases of children with posterior scleritis in their series of patients with this disease; Singh et al. ${ }^{5}$ included the case of a 14-year-old with posterior scleritis which resolved after treatment for 1 week with systemic steroids.

It is evident that there is a spectrum of presentation of posterior scleritis varying from mild cases which require little treatment to severe cases in which visual loss may develop from either optic atrophy or macular detachment. In this respect our series shows a surprising uniformity. The patients all presented with pain in the inflamed eye with lid swelling. Painful limitation of ocular movement was present in 4 of 5 cases. The disease took a protracted course and required long-term immunosuppression in all cases. In addition to this uniformity, it is also unusual that all 5 cases presented to this hospital over a period of 9 months when previously no case of posterior scleritis in children had been seen. This aroused the suspicion of an infectious aetiology, but there was nothing in the preceding medical history or evidence from serological investigation to suggest that this was indeed the case.

Optic disc swelling occurred in 4 of 5 cases with preservation of optic nerve function, and we believe that aggressive treatment may have prevented visual loss in all these eyes. Early diagnosis is essential to prevent irretrievable visual loss in this condition and suspicion must be aroused in children who present with a painful eye, lid swelling, limitation of ocular movement, optic disc swelling and serous detach- ment of the retina. Furthermore, the presence of an anterior uveitis that does not respond to topical therapy may indicate underlying posterior scleritis. The diagnosis may be made on the basis of B-scan ultrasonography, which showed scleral thickening in all 5 of our cases. However, scleral thickening can also be produced by acute pseudo-tumour of the orbit and for this reason we also performed CT scans of the orbit. ${ }^{2}$

All the cases in this series required high-dose immunosuppression to control the disease and prevent relapse. In 2 cases non-steroidal antiinflammatory agents had been tried without success. Immunosuppression with prednisolone alone was tried in all patients but in 4 cases it was not possible to reduce the dose below $30 \mathrm{mg}$ once daily without precipitating relapse, and in 2 cases the patients became overtly cushingoid and increased their body weight by at least a quarter. In one case prednisolone on alternate days was tried, to reduce the incidence of steroidal side effects, but this was not found to be as effective as a once daily dosage. In the cases requiring high-dose prednisolone and where medical complications intervened, cyclosporin $\mathrm{A}$ at a dose of $5 \mathrm{mg} / \mathrm{kg}$ was introduced as a steroid sparing agent. In all cases this second-line immunosuppression allowed the steroids to be reduced to a tolerable level for maintenance of therapy. In case 5 the drug needed to be stopped due to the development of proteinuria which was subsequently found to be due to a urinary tract infection. However, on re-introduction no further problems were found and thus cyclosporin $\mathrm{A}$ is both a useful and well-tolerated adjunct to prednisolone in children.

This study was supported in part by the Iris Fund for the Prevention of Blindness. We thank Mr P. Meyer for help in the management of case 5. We are indebted to Mrs D. Paterson for preparing the manuscript.

Key words: Children, Posterior scleritis.

\section{REFERENCES}

1. Calthorpe CM, Watson PG, McCartney ACE. Posterior scleritis: a clinical and histological survey. Eye 1988;2: 267-77.

2. Benson WE. Posterior scleritis. Survey Ophthalmol 1988;32:297-316.

3. Wald KJ, Spaide R, Patalano VJ, Sugin S, Yannuzzi LA. Posterior scleritis in children. Am J Ophthalmol 1992; 113:281-6.

4. Berger G, Reeser F. Retinal pigment epithelial detachments in posterior scleritis. Am J Ophthalmol 1989;90: 604-6.

5. Singh G, Guthoff R, Foster CS. Observations on long term follow-up of posterior scleritis. Am J Ophthalmol 1986; 101:570-5.

6. Chaques V, Lam S, Tessler HH, Mafee MF. Computed tomography and magnetic resonance imaging in the diagnosis of posterior scleritis. Ann Ophthalmol 1993; 25:89-94. 